\title{
PRODUCCIÓN DE BULBILLOS DE CEBOLLA OCAÑERA A PARTIR DE SEMILLA SEXUAL
}

\section{PRODUCTION OF OCAÑERA ONION BULBS FROM SEXUAL SEED}

\author{
Elibardo Pacheco Carrascala ${ }^{\mathrm{a}}$, José Arnoldo Granadillo Cuello ${ }^{\mathrm{b}}$ \\ ${ }^{a}$ Grupo GI@DS, Facultad de Ciencias Agrarias y del Ambiente, Universidad Francisco de Paula \\ Santander Ocaña, Colombia, elibardop@ hotmail.com \\ ${ }^{\mathrm{b}}$ Grupo GI@DS, Facultad de Ciencias Agrarias y del Ambiente, Universidad Francisco de Paula \\ Santander Ocaña, Colombia, jagranadilloc@ufpso.edu.co
}

Fecha de recepción: 12-05-2017

Fecha de aprobación: 22-06-2017

\begin{abstract}
Resumen: Con el sistema de manejo tradicionalista del cultivo de la cebolla en Ocaña, caracterizado por monocultivo, propagación por bulbos, altas proporciones de materia orgánica sin descomponer (gallinaza y bovinaza), y un manejo totalmente químico de las pestes; se favorecen prácticas que causan el degeneramiento progresivo del material de propagación (bulbos), con severas pudriciones fungosas y bacteriales; y a la vez alta contaminación de los suelos cebolleros. En esta investigación se planteó obtener bulbillos en semilleros a partir de semilla sexual, siendo estos pequeños, sanos y de buena calidad, que garantizan una buena cosecha. Se sembraron tres materiales: Hibrida Rosada Milenio F1; semilla Resaca de Hibrida Rosada milenio; y la variedad Red Creole. Como resultado se observa, que el material Hibrida Rosada Milenio F1, produce bulbillos compactos y de buena calidad a los 52 días en dos ensayos. La semilla del material Resaca y la variedad Red Creole, no llenaron bulbo y la planta continúa produciendo hojas a pesar de esperar hasta los 100 días; y los pocos bulbos formados no son compactos, y al final del almacenamiento (45 días) aparecen de mala calidad.
\end{abstract}

Palabras clave: bulbillos de cebolla, semilla sexual, propagación asexual

\begin{abstract}
With the system of traditionalist management of onion cultivation in Ocaña, characterized by monoculture, propagation by bulbs, high proportions of organic matter without decomposing (chicken and cattle), and a totally chemical management of pests; Favors practices that cause progressive degeneration of propagation material (bulbs), with severe fungal and
\end{abstract}


bacterial decay; And at the same time high contamination of the cebolleros floors. In this research it was proposed to obtain bulbillos in seedlings from sexual seed, these being small, healthy and of good quality, that guarantee a good harvest. Three materials were planted: Hibrida Rosada Milenio F1; Seed Resaca Hybrid Rose; And the Red Creole variety. As a result, it can be observed that the material Hibrida Rosada Milenio F1 produces compact bulbs of good quality at 52 days in two tests. The seed of the material Resaca and the variety Red Creole, did not fill bulbs and the plant continues to produce leaves despite waiting up to 100 days; And the few bulbs formed are not compact, and at the end of storage (45 days) appear poor quality.

Keywords: Onion bulb, sexual seed, asexual propagation

\section{INTRODUCCIÓN}

El cultivo de la cebolla ocañera, era la principal actividad agrícola de la Provincia de Ocaña. Actualmente existen 7.080 pequeños productores, que siembran alrededor de 3.000 Has, en los Municipios de Ocaña, Abrego, La Playa de Belén, San Calixto, El Carmen y Villa Caro en el departamento de Norte de Santander; González y Río de Oro, en el Sur del Cesar (Duque, Perdomo y Jaramillo, 1983; Corpoica, 2003). La producción departamental de cebolla ocañera aumentó entre 2003 y 2006 un 38\%, sin embargo entre el 2006 y el 2014 sufrió un descenso del 83\% (ENA, 2014) debido a la utilización de bulbos de mala calidad y al desarrollo de enfermedades.

El sistema de propagación de la cebolla, usado en la región de Ocaña, es bulbos, dejados de cosechas anteriores (Pinzón, 2009; Aguirre y Piraneque, 2013; Granadillo y Trigos, 2016) o comprados en fincas vecinas; sin ninguna técnica de selección, ni mejoramiento, y sembrados en el mismo lote durante muchos años; ocasionando esto un degeneramiento progresivo de los bulbos y una alta infestación de microorganismos fungosos y bacteriales, como: Fusarium sp, Pyrenochaeta terrestris, Eruwinia carotovora, Pseudomonas cepaceae; que producen pudriciones radiculares, de los bulbos, y que se difunde rápidamente a todos los suelos cebolleros (Cuello y Carrascal, 2014). Por otro lado la utilización tradicional de bulbos para su reproducción ha ocasionado androesterilidad, o incapacidad para producir gametos viables (Mejía López y Jaramillo Henao, 1996; Ramírez, 2006; Mallor Giménez \& Garcés Claver, 2015). Sin embargo existe semilla sexual en el mercado de algunos híbridos de cebolla ocañera que pueden ayudar a disminuir la utilización de bulbos, la incidencia de enfermedades y a mejorar su producción, como el Hibrido Rosada Milenio F1, Hibrido Rosada Mileno Resaca y Red creole.

Como alternativa de control los agricultores aumentan las frecuencias $y$ dosis de pesticidas, incrementando drásticamente los costos de producción; y con las reducidas producciones por unidad de área que se obtienen, el cultivo se torna no rentable, cada día se desplaza su siembra a zonas más altas en las cordilleras (Pacheco, Bohórquez y Vergel, 2007).

El objetivo de esta investigación fue comparar la producción de bulbos en tres variedades de cebolla roja, Hibrido Rosada Milenio F1, Hibrido Rosada Mileno Resaca y Red creole, para la obtención de bulbillos sanos y de tamaño adecuado a partir de semilla sexual, mejorando así la producción 
y rentabilidad de este cultivo en la zona y para Colombia.

\section{METODOLOGÍA}

La técnica consiste en hacer el semillero con la semilla de la cebolla y dejar que produzca llenado de bulbillos en el sitio; una vez maduros, se cosecha y se lleva a reposo durante 40 a 45 días, para luego ser sembrados en el campo y allí obtener bulbos comerciales (Pacheco, Manzano y Buitrago, 1998). Esto se realizó en tres pasos:

Primero: Se sembraron los tres materiales: Hibrida Rosada Milenio F1; semilla Resaca de Hibrida Rosada Milenio y la variedad Red Creole. Se hizo la prueba de germinación (Figura 1) de cada material y luego se sembraron en eras, aplicando abono fermentado tipo Bocashi (Figura 2), y se instaló el riego por aspersión (Figura 3).

Se empleó un diseño experimental completamente al azar, con tres tratamientos y nueve repeticiones. Cada unidad experimental constó de una era de 10 metros cuadrados cada una. Sobre cada era se aplicó 100 gramos de semillas, esta etapa se realizó en la granja experimental de la Universidad Francisco de Paula Santander. La variable medida fue producción de bulbillos en $\mathrm{Kg}$.
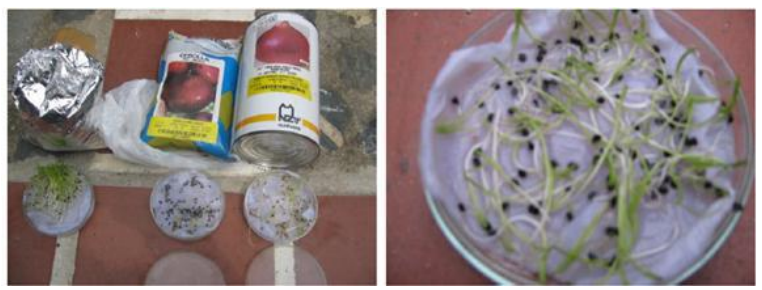

Figura 1. Pruebas de germinación de semillas de cebolla. Fuente: Autores del proyecto.

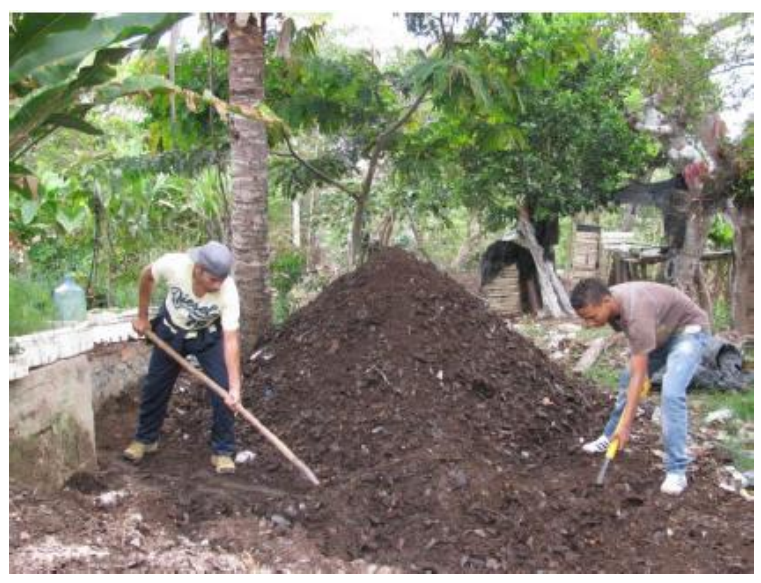

Figura 2. Preparación del Bocashi. Fuente: Autores del proyecto.

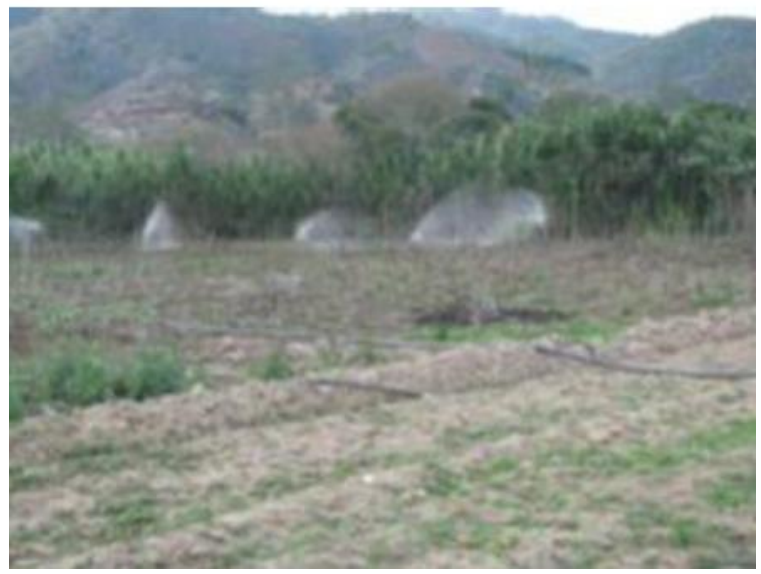

Figura 3. Sistema de riego implementado. Fuente: Autores del proyecto.

Segundo: Con la semilla Resaca se probaron dos densidades de siembra: 50 y 75 gramos por era, con siete replicas (eras) por cada una, para un total de 14 unidades experimentales. La variable medida fue producción de bulbillos en $\mathrm{Kg}$.

Tercero: Con el material Hibrida Rosada Milenio F1, se ensayaron tres densidades de siembra $\left(7.5,10\right.$ y $\left.15 \mathrm{gr} / \mathrm{m}^{2}\right)$; este semillero se hizo en el corregimiento de la Ermita, en el municipio de Ocaña, Norte de Santander. Cada unidad experimental fue de $1 \mathrm{~m}^{2}$ y se hicieron 10 repeticiones de cada una.

Además se determinó el tiempo (días) para iniciar formación de bulbos. 


\section{RESULTADOS}

\subsection{Producción de bulbillos}

De la siembra de los tres materiales de cebolla: Hibrida rosada milenio F1, Hibrida rosada milenio resaca y Red creole se obtuvieron los siguientes resultados:

Para medir la cosecha se tomó la producción en $\mathrm{Kg}$ de $5 \mathrm{~m}^{2} / \mathrm{era}$.

\begin{tabular}{|c|c|c|}
\hline HRM F1 & HRM RESACA & RED CREOLE \\
\hline 6,3 & 3,3 & 2,0 \\
\hline 6,9 & 2,0 & 1,0 \\
\hline 6,3 & 1,5 & 0,8 \\
\hline 2,0 & 2,6 & 3,0 \\
\hline 5,0 & 1,5 & 1,0 \\
\hline 3,5 & 6,0 & 1,0 \\
\hline 2,5 & 0,8 & 2,0 \\
\hline 3,15 & 0,5 & 1,0 \\
\hline 3,9 & 0,5 & 1,0 \\
\hline
\end{tabular}

Tabla 1. Producción de bulbillos en $\mathrm{Kg}$, por cada una de las variedades de cebolla ensayadas. Fuente: Autores del proyecto.

\begin{tabular}{|l|l|l|l|l|l|}
\hline Variable & Media & E. E. & Desv.Est. & Varianza & CoefVar \\
\hline HRM F1 & 4,394 & 0,599 & 1,797 & 3,228 & 40,88 \\
\hline $\begin{array}{l}\text { HRM } \\
\text { RESACA }\end{array}$ & 2,078 & 0,583 & 1,748 & 3,054 & 84,11 \\
\hline $\begin{array}{l}\text { RED } \\
\text { CREOLE }\end{array}$ & 1,422 & 0,248 & 0,745 & 0,554 & 52,36 \\
\hline
\end{tabular}

Tabla 2. Estadística descriptiva de la producción de bulbillos en $\mathrm{Kg}$, para las variedades de cebolla ensayadas. Fuente: Autores del proyecto.

La producción media de bulbillos en Kg más alta correspondió a la variedad Hibrida Rosada Milenio F1 con 4,394 Kg por era (Tabla 2; Figura 4).

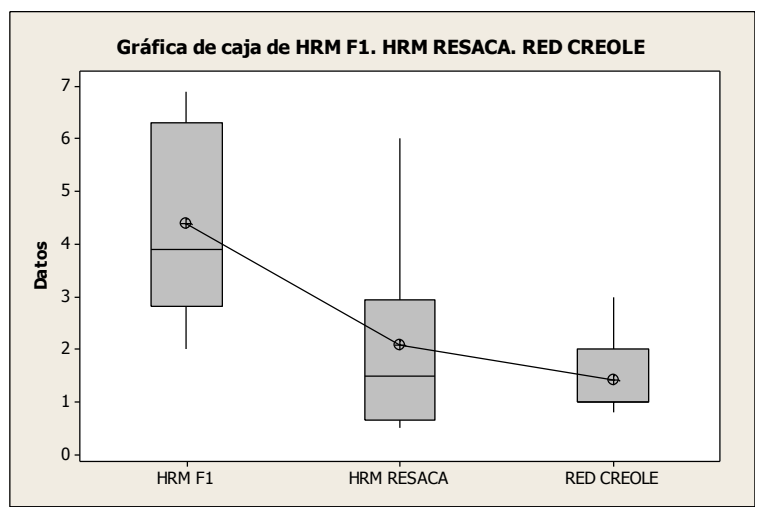

Figura 4. Grafica de cajas, que nos muestra las medias de producción de bulbillos en $\mathrm{Kg}$ para cada una de las variedades ensayadas. Fuente: Autores del proyecto.

Al realizar el análisis de varianza se pudo observar que el $p$ valor es menor que el nivel de confianza propuesto 0,05 señalando que hay diferencia significativa entre las medias de la variable producción de bulbillos en kilogramos (Tabla 3). La prueba de Tukey arrojó que existe diferencia significativa entre HRM F1 y las otras dos variedades, no así entre HRM Resaca y Red Creole.

\begin{tabular}{|l|l|l|l|l|l|}
\hline Fuente & GL & SC & MC & F & P \\
\hline Factor & 2 & 43,89 & 21,95 & 9,63 & 0,001 \\
\hline Error & 24 & 54,69 & 2,28 & & \\
\hline Total & 26 & 98,59 & & & \\
\hline $\begin{array}{l}\text { S }=1,510 \text { R-cuad. }=44,52 \% \\
\text { R-cuad. (ajustado) }=39,90 \%\end{array}$ \\
\hline
\end{tabular}

Tabla 3. Análisis de varianza para la producción de bulbillos en $\mathrm{Kg}$, para las variedades de cebolla ensayadas. Fuente: Autores del proyecto.

Adicionalmente, se observó que el material Hibrido Rosada Milenio F1, inicio formación de bulbos a los 52 días después de la siembra, y al momento de la cosecha todas las plantas habían llenado completamente el bulbillo siendo este compacto y redondeado; mientras que los demás materiales sembrados a pesar de que iniciaron el llenado de bulbo a los 52 días, las plantas siguieron produciendo hojas nuevas y se detuvo el llenado de bulbo en la mayoría de las plántulas; esto posiblemente debido al cambio climático que se presentó 
por la iniciación de las lluvias. Este último material fue cosechado pero los bulbos evidenciaron mala calidad y consistencia esponjosa no apto para su siembra (Figuras $5,6$ у 7$)$.

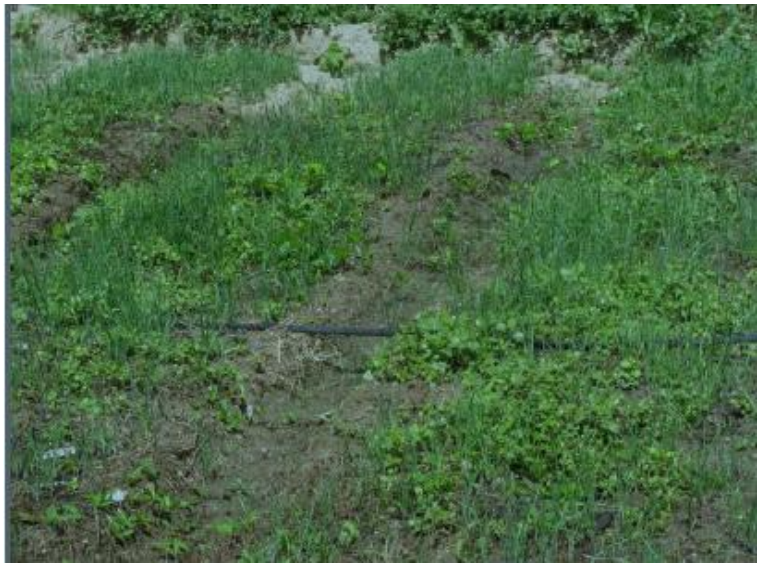

Figura 5. Desarrollo del cultivo. Fuente: Autores del proyecto.

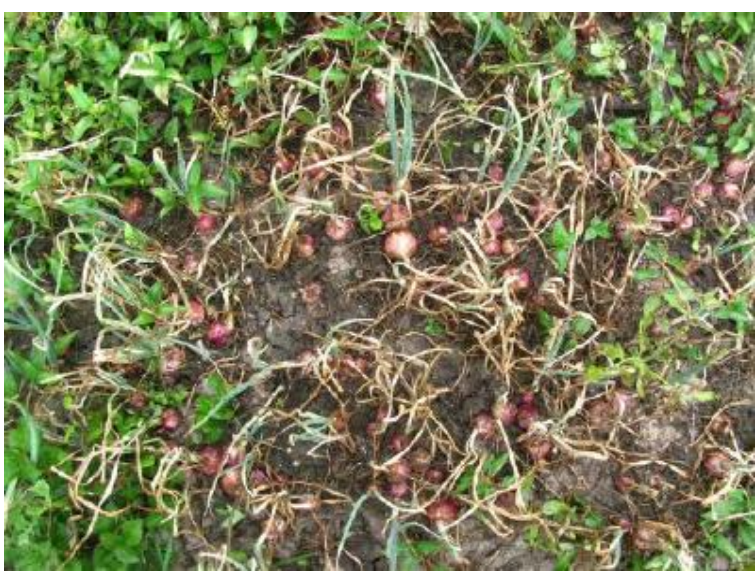

Figura 6. Desarrollo de bulbos. Fuente: Autores del proyecto

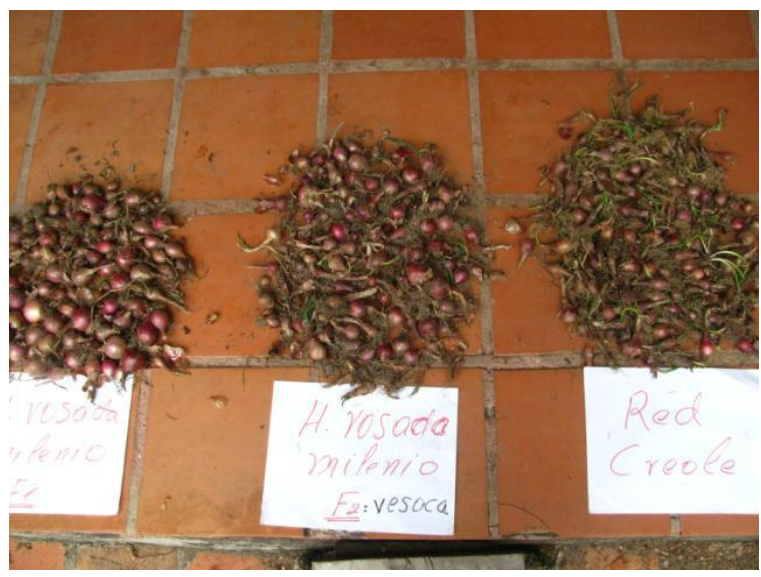

Figura 7. Cosecha de bulbos de las tres variedades de cebolla ensayadas. Fuente: Autores del proyecto.

\subsection{Densidades de siembra y producción de bulbillos con HRM Resaca}

La variedad HRM Resaca fue sembrada con densidades de 50 y 75 gr/era (Tabla 4). Para la cosecha se tomaron en cuenta $5 \mathrm{~m}^{2}$ de cada era. La producción media de bulbillos más alta se observó en la densidad de 75 gr/era con promedio de 4,371 $\mathrm{Kg}$ por era (Tabla 5). A pesar de que el inicio del llenado de los bulbos ocurrió entre los 52 y los 60 días, con el cambio de clima por la entrada del invierno este se detuvo para dar paso a la producción de nuevas hojas, ocurriendo un macollamiento de la planta, y a pesar de esperar hasta los 90 días, la mayoría de plantas no formaron bulbillos, y los que se formaron resultaron de mala calidad.

\begin{tabular}{|c|c|}
\hline D 50 g/10 m2 & D 75 g/10 m2 \\
\hline 2,7 & 4,2 \\
\hline 3,9 & 3,0 \\
\hline 4,1 & 3,9 \\
\hline 2,5 & 4,6 \\
\hline 4,0 & 5,9 \\
\hline 2,5 & 4,0 \\
\hline 2,7 & 5,0 \\
\hline
\end{tabular}

Tabla 4. Producción de bulbillos en $\mathrm{Kg}$ con las dos densidades ensayadas, para la variedad HRM Resaca. Fuente: Autores del proyecto. 


\begin{tabular}{|l|l|l|l|l|l|}
\hline Variable & Media & E. E. & Desv.Est. & Varianza & CoefVar \\
\hline $\begin{array}{l}\text { D } 50 \\
\text { g/era }\end{array}$ & 3,200 & 0,285 & 0,755 & 0,570 & 23,59 \\
\hline $\begin{array}{l}\text { D } 75 \\
\text { g/era }\end{array}$ & 4,371 & 0,347 & 0,918 & 0,842 & 21,00 \\
\hline
\end{tabular}

Tabla 5. Estadística descriptiva de la producción de bulbillos en $\mathrm{Kg}$, en dos densidades de siembra de la variedad de cebolla HRM Resaca. Fuente: Autores del proyecto.

Al realizar el análisis de varianza se pudo observar que el $p$ valor es menor que el nivel de confianza propuesto 0,05 señalando que hay diferencia significativa entre las medias de la variable producción de bulbillos en kilogramos de las dos densidades de siembra utilizadas (Tabla 6). La prueba de Tukey arrojó que existe diferencia significativa entre las dos densidades de siembra.

\begin{tabular}{|l|l|l|l|l|l|}
\hline Fuente & GL & SC & MC & F & P \\
\hline Factor & 1 & 4,80 & 4,803 & 6,80 & 0,023 \\
\hline Error & 12 & 8,47 & 0,706 & & \\
\hline Total & 13 & 13,27 & & & \\
\hline $\begin{array}{l}\text { S }=0,8404 \text { R-cuad. }=36,17 \% \\
\text { R-cuad. (ajustado) }=30,86 \%\end{array}$ \\
\hline
\end{tabular}

Tabla 6. Análisis de varianza para la producción de bulbillos en $\mathrm{Kg}$, para la variedad HRM Resaca. Fuente: Autores del proyecto.

\subsection{Densidades de siembra con Hibrida Rosada Milenio F1}

La variedad HRM F1 fue sembrada con densidades de 5, 7,5 y $15 \mathrm{gr} / \mathrm{m}^{2}$ (Tabla 7). Para la cosecha se tomó en cuenta $1 \mathrm{~m}^{2}$. La producción media de bulbillos más alta se observó en la densidad de $15 \mathrm{gr} / \mathrm{m}^{2}$ con promedio de 2,250 $\mathrm{Kg}$ por $\mathrm{m}^{2}$ (Tabla 8). Esta variedad inició llenado de bulbo a los 52 días y a los 72 días, presentó bulbillos compactos y con el follaje maduro en algunas plantas y seco en la mayoría, siendo necesario cosecharlas. Este hibrido mostró un buen comportamiento agronómico y buena calidad de los bulbos.

\begin{tabular}{|c|c|c|}
\hline $\mathbf{5} \mathbf{~ g r} / \mathbf{m}^{\mathbf{2}}$ & $\mathbf{7 . 5} \mathbf{~ g r} / \mathbf{m}^{\mathbf{2}}$ & $\mathbf{1 5} \mathbf{~ g r} / \mathbf{m}^{\mathbf{2}}$ \\
\hline 1.2 & 1,8 & 1,8 \\
\hline 1,8 & 1,8 & 1,5 \\
\hline 1,2 & 2,5 & 2,4 \\
\hline 1,5 & 2,9 & 3,1 \\
\hline 1,4 & 2,3 & 2,5 \\
\hline 2,5 & 2,0 & 2,6 \\
\hline 1,5 & 1,6 & 1,8 \\
\hline 1,7 & 1,5 & 2,0 \\
\hline 1,6 & 1,5 & 2,5 \\
\hline 2,5 & 1,6 & 2,3 \\
\hline
\end{tabular}

Tabla 7. Producción de bulbillos en $\mathrm{Kg}$ con diferentes densidades de siembra. Fuente: Autores del proyecto.

Estos bulbillos son compactos y de tamaño pequeño, ideales para obtener al sembrarlos bulbos de tamaño adecuado para los mercados de la Costa Atlántica.

\begin{tabular}{|l|l|l|l|l|l|}
\hline Variable & Media & E. E. & Desv.Est. & Varianza & CoefVar \\
\hline $5 \mathrm{gr} / \mathrm{m}^{2}$ & 1,690 & 0,148 & 0,468 & 0,219 & 27,68 \\
\hline $7,5 \mathrm{gr} / \mathrm{m}^{2}$ & 1,950 & 0,150 & 0,474 & 0,225 & 24,33 \\
\hline $15 \mathrm{gr} / \mathrm{m}^{2}$ & 2,250 & 0,150 & 0,474 & 0,225 & 21,08 \\
\hline
\end{tabular}

Tabla 8. Estadística descriptiva de la producción de bulbillos en $\mathrm{Kg}$, en tres densidades de siembra de la variedad de cebolla HRM F1. Fuente: Autores del proyecto.

\begin{tabular}{|l|l|l|l|l|l|}
\hline Fuente & GL & SC & MC & F & P \\
\hline Factor & 2 & 1,571 & 0,785 & $\mathbf{Z}^{\prime}, 52$ & 0,044 \\
\hline Error & 27 & 6,019 & 0,223 & & \\
\hline Total & 29 & 7,590 & & & \\
\hline $\begin{array}{l}\text { S }=0,4722 \text { R-cuad. }=20,69 \% \\
\text { R-cuad. (ajustado) }=14,82 \%\end{array}$ \\
\hline
\end{tabular}

Tabla 9. Análisis de varianza para la producción de bulbillos en Kg, para la variedad HRM F1. Fuente: Autores del proyecto.

Al realizar el análisis de varianza se pudo observar que el $p$ valor es levemente menor que el nivel de confianza propuesto 0,05 señalando que podría haber diferencia significativa entre al menos dos de las medias de la variable producción de bulbillos en kilogramos de las tres 
densidades de siembra utilizadas (Tabla 9). La prueba de Tukey arrojó que existe diferencia significativa solo entre las densidades de siembra de $5 \mathrm{gr} / \mathrm{m}^{2}$ y $15 \mathrm{gr} / \mathrm{m}^{2}$, en esta última se registró la mayor cantidad de kilogramos producidos por metro cuadrado.

\section{DISCUSIÓN}

La variedad de cebolla Hibrida Rosada Milenio F1 tuvo una mayor producción de bulbos en comparación con las variedades Hibrida Rosada Milenio Resaca y la Red Creole. A pesar de que las tres variedades iniciaron formación de bulbos a los 52 días después de la siembra, solo en la muestra de la variedad HRM F1 todas las plantas habían llenado completamente el bulbillo en el momento de la cosecha, siendo este además compacto y redondeado, características esenciales de los bulbos de buena calidad.

En la variedad HRM Resaca y Red Creole se observó el mal llenado de los bulbos, lo cual ocurrió debido al cambio climático, no siendo así para la variedad HRM F1. Sin embargo Pinzón, Machado y Báez (2006) también confirman la influencia de factores como la temperatura y la humedad relativa en el proceso de bulbificación.

Al probar diferentes densidades de siembra para las variedades HRM F1 y HRM Resaca Se observó que a mayor densidad de siembra hay mayor número de bulbillos, pero de menor tamaño, esto debido a la competencia por el alimento, presentándose gran cantidad de bulbillos miniatura. Como afirma Pinzón, Machado y Báez (2006); Medina (2008), sembrar cebolla en bajas densidades produce bajos rendimientos porque se desarrolla mayor cantidad de bulbos medianos y grandes, mientras que su siembra en altas densidades permite el desarrollo de mayor cantidad de bulbos pequeños.

\section{CONCLUSIONES}

En la variedad Hibrido Rosada Milenio f1 se observó mayor rendimiento en la producción de bulbos bajo las condiciones de este ensayo obteniendo bulbos de buena calidad agronómica.

Se observó que a mayor densidad de siembra aumenta la cantidad de bulbos producidos, sin embargo estos son de tamaño pequeño, debido al fenómeno de competencia que obliga a la producción de biomasa a partir de una cantidad limitada de nutrientes.

A través de este ensayo se puede mejorar la producción y rentabilidad del cultivo de cebolla Ocañera en la zona y para Colombia.

\section{BIBLIOGRAFÍA}

Aguirre, F. S. E. y Piraneque, G. N. V. (2013). Horticultura. Contenido didáctico del curso viveros. Escuela de Ciencias Agrícolas, Pecuarias y del medio Ambiente. UNAD, Bogotá, 241p.

CORPOICA, ACCA, PRONATTA. 2003. Manejo de Riego en cebolla ocañera. Manual Técnico.

Cuello, J. A. G., \& Carrascal, E. P. (2014). Desinfección para el establecimiento in vitro de cebolla ocañera Allium cepa L. Revista Ingenio UFPSO, 7(1), 123-131.

Duque, M., Perdomo, C. M., \& CE Jaramillo, J. (1983). Crecimiento y 
absorción de nitrógeno, fósforo y potasio en cebolla, Allium cepa L.: variedad ocañera. Acta Agronómica (Colombia) v. 33 (4) p. 16-22ISSN 0120-2812.

Encuesta Nacional Agropecuaria ENA 2014. (2015). Boletín técnico. Departamento Nacional de Estadística DANE. Bogotá D.C. Recuperado de: http://www.dane.gov.co/files/investi gaciones/agropecuario/enda/ena/201 4/boletin_ena_2014.pdf.

Granadillo, C. J. A., Trigos, T. M. H. (2016). Establecimiento in vitro de la cebolla ocañera Allium cepa L. para la producción de semilla asexual limpia en la provincia de Ocaña, Norte de Santander. Trabajo de grado de Especialización. Universidad Nacional Abierta y a Distancia. Bogotá Colombia.

Mallor Giménez, C., \& Garcés Claver, A. (2015). La androesterilidad en cebolla (Allium cepa L.). Horticultura, 318, p. 28-31.

Medina, J. (2008). Cebolla: guía técnica. Instituto Dominicano de Investigaciones Agropecuarias $y$ Forestales (IDIAF). Santo Domingo, DO. $64 p$.

Mejía López, J., \& Jaramillo Henao, D. (1996). Cebolla ocañera. El cultivo del ajo y las cebollas en Colombia, 43-59.

PACHECO C. E., BOHORQUEZ, E.; VERGEL, M. (2007). Caracterización del cultivo de la cebolla en la Provincia de Ocaña. Tesis de Especialista en Estadística Aplicada. Universidad Francisco de
Paula Santander Ocaña. Ocaña, Norte de Santander.

PACHECO C. E., MANZANO, I., BUITRAGO, W. (1988). Evaluación de Sistemas de Propagación de cebolla cabezona. Tesis Tecnólogo Agropecuario. Universidad Francisco de Paula Santander. Facultad de Ciencias Agrarias. Tecnología Agropecuaria. Ocaña, Norte de Santander.

Pinzón, R. (2009). Los cultivos de cebolla y ajo en Colombia: estado del arte y perspectivas. Revista Colombiana de Ciencias Hortícolas, 3(1).

Pinzón Ramírez, H., Ospina Machado, J. E., Báez, A. (2006). Producción y tecnologías de curado y almacenamiento de cebolla de bulbo. CORPOICA, Bogotá, 36p (ES).

Ramírez, L. (2006). Utilización de la androesterilidad para la producción de semilla híbrida. Cátedra de producción vegetal genética y mejora vegetal. Departamento de Producción Agraria, Universidad Pública de Navarra. Pamplona, España. 